\title{
Substituição Completa da Aorta Ascendente e da Valva Aórtica com Tubo Valvulado de Pericárdio Bovino
}

Robert V. ARDITO*, José L. Verde SANTOS, Rita C. MAYORQUIM, Oswaldo T. GRECO, Marcos ZAIANTCHIC, Henry G. SOTO, José Luiz B. JACOB, Domingo M. BRAILE

RBCCV 44205-33

ARDITO, R. V.; SANTOS, J. L. V.; MAYORQUIM, R. C.; GRECO, O. T; ZAIATCHICK, M.; SOTO, H. G.; JACOB, J. L. B.; BRAILE, D. M. - Substituição completa da aorta ascendente e da valva aórtica com tubo valvulado de pericárdio bovino. Rev. Bras. Cir. Cardiovasc., 2 (2): 129-138, 1987.

RESUMO: Os autores fazem uma revisão da técnica cirúrgica empregada para o tratamento de aneurismas da aorta ascendente associados a lesōes da valva aórtica. Apresentam resultados obtidos com 11 (onze) paciente operados pela técnica de BENTALL e DE BONO, com enxerto tubular valvulado aberto de pericárdio bovino IMC, sem mortalidade devida à técnica ou ao enxerto utilizado. Desses pacientes, 8 eram do sexo masculino, com idade variando entre 31 e 65 anos, média de 50 anos; 3 eram do sexo feminino, com idade entre 20 e 53 anos, média de 38 anos. Duas cirurgias foram realizadas, em regime de urgência, e 9 de emergência. O tubo de pericárdio bovino utilizado foi de n: 23 em 1 caso, n: 25 em 2, n: 29 em 6 e n: 31 em 2 casos. Dois pacientes tiveram lesão mitral associada com troca valvar (prótese biológica IMC). Um paciente necessitou de ponte de veia safena para coronária direita, por dissecçāo do óstio coronário. O diagnóstico histopatológico mostrou: degeneração mixomatosa em 4 casos, fibrose em 4 casos, doença reumática cicatrizada em 2 casos e pancardite em apenas 1 caso. Após discorrerem sobre dificuldades e complicaçōes do método, concluem que a técnica utilizada é a de escolha no tratamento da referida patologia e que o tubo valvulado de pericárdio bovino facilita o ato cirúrgico, por sua alta flexibilidade e por ser altamente hemostático, não necessitando de medidas pré-coagulativas.

DESCRITORES: aorta ascendente, cirurgia; valva aórtica, cirurgia; tubo valvulado, pericárdio bovino.

\section{INTRODUÇĀO}

O aneurisma da aorta ascendente, bem como a dilatação ânulo-aórtica com dilatação pronunciada da porção ascendente do arco aórtico, tem sido alvo de um substancial avanço terapêutico-cirúrgico, desde 1952 , com COOLEY \& De BAKEY ${ }^{4}$ e COOLEY et alii ${ }^{5}$, que estabeleceram que o aneurisma da aorta torácica è uma doença fatal, comparada com o câncer, enfatizando a necessidade de uma intervençāo agressiva, removendo a doença, bem como a reconstrução do vaso, quando possivel.
A circulação extracorpórea (CEC) foi o que realmente possibilitou a cirurgia de aneurismas, removendo-os acima das coronárias, interpondo-se enxerto homólogo ${ }^{4}$. Mais tarde, enxertos sintéticos ${ }^{5}$ foram propostos e, até hoje, são empregados com sucesso por vários autores, com diferentes técnicas (GROVES et alii ${ }^{9}$ ). Observou-se que os aneurismas sảo causados pela dilatação do anel da valva aórtica com grande regurgitação. Isto ocorre muito rapidamente, tornando-se um fator predominante para a intervenção cirúrgica.

- Do Instituto de Moléstias Cardiovasculares.

Endereço para separatas: Roberto V. Ardito. Rua Castelo d'Água, 3030. 15015 São José do Rio Preto, SP. Brasil. 
ARDITO, R. V.: SANTOS, J. L. V.; MAYORQUIM, R. C.: GRECO, O. T: ZAIATCHICK, M.: SOTO, H. G.: JACOB. J. L. B.: BRAILE. D. M. - Substituiçâo completa da aorta ascendente e da valva aortica com tubo valvulado de pericardio bovino. Rev. Bras. Cir. Cardiovasc. 2(2): 129-138, 1987.

O tratamento dos aneurismas da aorta ascendente. associados com doença valvar, têm permanecido como técnica formidável e desafiadora, devido à grande redução da mortalidade cirúrgica. BENTALL \& De BONO ! SINGH \& BENTALL ${ }^{13}$, EDWARDS \& KERR ${ }^{8}$ descreveram as técnicas para substituição completa da aorta ascendente, com tubo valvulado e reimplante dos óstios coronários.

O presente estudo, realizado com 11 doentes portadores de aneurisma da aorta ascendente e insuficiência da valva aórtica, mostra a experiência com enxerto tubular valvulado de pericárdio bovino aberto, para facilitar o implante dos óstios coronários.

O pericárdio bovino foi empregado, em nosso Serviço, pela primeira vez, em 1979, para reconstrução da aorta, onde propúnhamos o alargamento da raiz da aorta com patch de pericárdio bovino tratado com glutaraldeido e conservado em formaldeído, pela técnica descrita por MANOUQUIAN \& SEYBOLD-EPTING ${ }^{11}$ e modificada por BRAILE et alii ${ }^{3}$.

\section{TÉCNICA CIRÚRGICA}

O coração é exposto por esternotomia mediana e instalada CEC, após heparinização, com canulação individual das cavas superior e inferior. O retorno é feito pela aorta, quando a porção mais distal é normal, ou pela artéria femoral. Utilizamos oxigenador de bolhas modelo "Harvey" com fluxo de $2.4\left(1 / \mathrm{m}^{2}\right)$ de superfície corporal. Obtivemos hipotermia moderada $\left(25\right.$ a $\left.28^{\circ} \mathrm{C}\right)$ medida pela orofaringe. Após iniciada a perfusão, a aorta é pinçada o mais distalmente possível, onde, na maioria dos casos, apresenta normalidades de suas paredes. O aneurisma é aberto e cardioplegia é feita diretamente nas coronárias, até a temperatura miocárdica atingir aproximadamente $8^{\circ} \mathrm{C}$, com volume entre 800 e 1000 $\mathrm{ml}$ da solução. $\mathrm{O}$ anel valvar está invariavelmente dilatado e as cúspides, geralmente, normais, principalmente na síndrome de Marfan; em outros casos, trata-se de valva aórtica bicúspide com calcificação intensa. Após a retirada das lascíneas, são passados pontos em $U$ no anel, do ventrículo para a aorta, o mais próximo possivel, para evitar vazamentos, que seriam extremamente difíceis de correção (Figura 1B). Após a passagem de pontos no anel aórtico, inicia-se a passagem de pontos no anel do tubo valvulado, de forma que a abertura permaneça voltada para cima. Os pontos são amarrados normalmente (Figura $1 \mathrm{C}$ ). Após a implantação do tubo no anel aórtico, são feitas duas aberturas neste, onde serão anastomosados os óstios coronários, separadamente, com fios de Prolene $5 / 0$ e sutura contínua (Figura 1D, E, F, G). As marcas dos locais onde serâo implantados os óstios são facilitadas pela abertura apresentada pelo tubo. Devemos lembrar que a solução cardioplégica é repetida a cada 20 minutos, mantendo a temperatura miocárdica abaixo de $15^{\circ} \mathrm{C}^{2}$. O próximo passo é anastomosar o tubo valvulado distalmente à aorta, após feita a medida, respeitando a curvatura do arco aórtico (posteriormente mais curto que anteriormente). A anastomose distal é realizada com fios de Prolene $4 / 0$ e feita sutura continua com pontos bem próximos (Figura $1 \mathrm{H}$ ). Somente agora o tubo valvulado é fechado à sua frente. com pontos de Prolene $5 / 0$ em sutura contínua (Figura 11). A retirada do ar é feita através de bolsa feita na parede lateral do tubo, ou em região da aorta normal distal. Em seguida, é iniciada a reperfusão com sangue quente, por derivação da linha arterial, com solução de papaverina e glicose, com a aorta pinçada e a pressâo controlada na raiz. A aorta é despinçada, após o coração ter assumido ritmo sinusal, com pressão de átrio esquerdo monitorada. O sangramento pelo enxerto, que sempre ocorre neste tipo de cirúrgia, nāo acontece neste caso, já que o pericárdio bovino preservado é impermeável e de fácil sutura. O restante da aorta aneurismática é suturado, sobre o enxerto, ou não (Figura 11, J). Após a retirada de CEC e reposição de volemia, é feita a antagonização da heparina com protamina na proporção de 1:1.3, monitorada pelo tempo ativado de coagulação (TAC) com celite. Devemos salientar que o fato de suturar a aorta sobre o enxerto às vezes provoca sangramentos por distorçōes das anastomoses, como apregoado por alguns autores.

\section{MATERIAL}

O tubo valvulado é constituido de uma válvula de pericárdio bovino no seu interior, que tem características especiais, quanto à confecção de sua aba, onde é suturado um tubo de pericárdio bovino também tratado pelos mesmos meios químicos da válvula.

Devemos realçar que o tubo é constituído por um patch de pericárdio com $11 \mathrm{~cm}$ de comprimento e terá o seu diâmetro determinado pelo tamanho da válvula e, sobretudo, apresenta-se, na sua porção próxima à válvula, mais dilatado, para facilitar as anastomoses com os óstios coronários.

O tubo apresenta-se suturado na aba da válvula, por dentro do Dacron, o que confere, além da firmeza, uma impermeabilizaçāo, evitando sangramento; contudo, o tubo valvulado só apresenta uma pequena porção suturada, no sentido longitudinal, de $\pm 1,5 \mathrm{~cm}$ e 0 restante permanece aberto totalmente, para facilitar as anastomoses coronárias, reinfusōes de cardioplegia e medida adequada do tubo na sua porção mais distal.

Este tubo valvulado foi desenvolvido nos laboratórios de pesquisa da IMC Biomédica, em São José do Rio Preto. 
ARDITO. R. V.: SANTOS. J. L. V.: MAYORQUIM. R. C.: GRECO. O. T: ZAIATCHICK. M.: SOTO. H. G.: JACOB. J. L. B.: BRAILE.

D. M. - Substituição completa da aorta ascendente e da valva aortica com tubo valvulado de pericardio bovino. Rev. Bras.

Cir. Cardiovasc. 2(2): 129-138, 1987

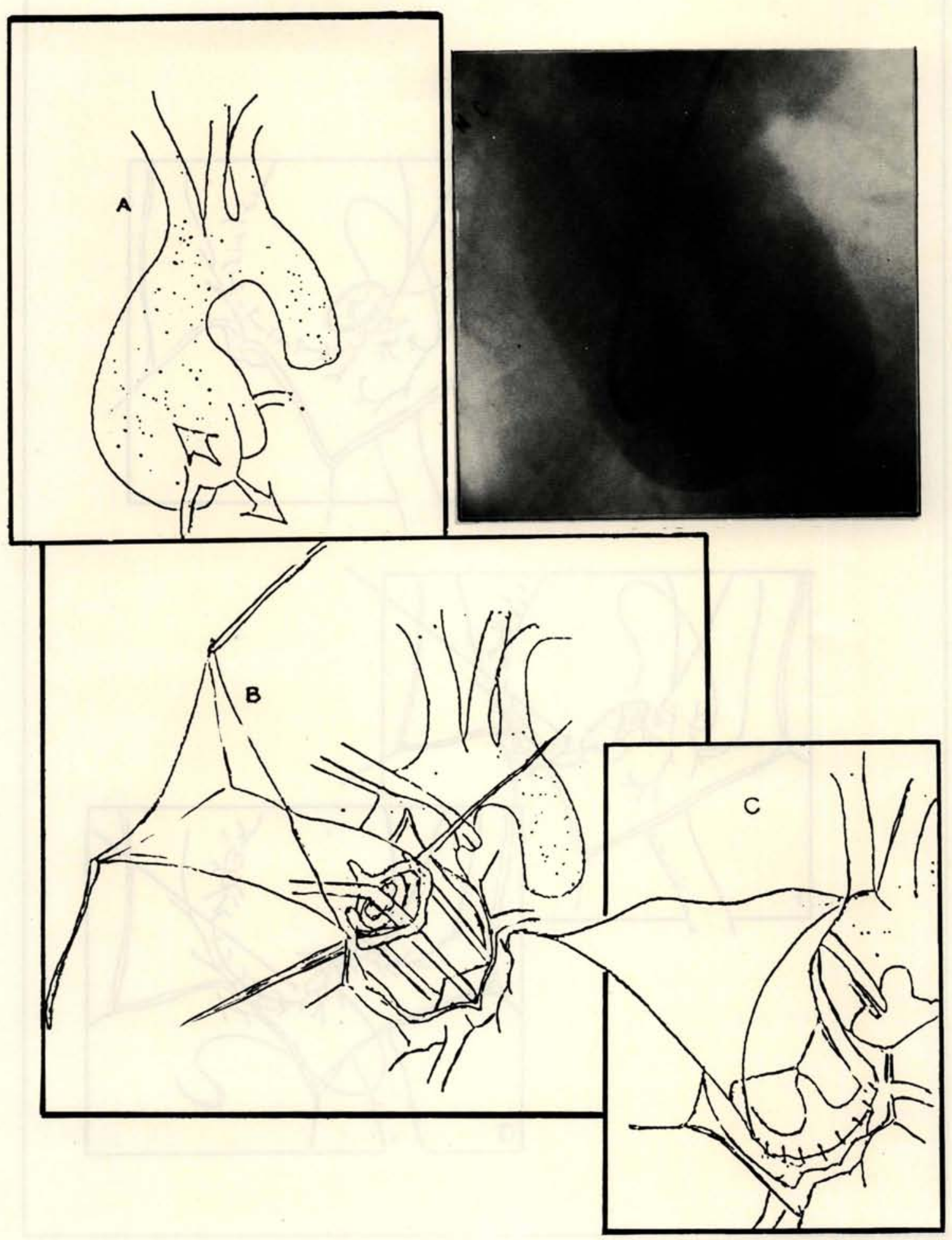

Fig. 1 - A, B, C. 
ARDITO, R. V : SANTOS, J. L. V.: MAYORQUIM. R. C.: GRECO. O. T: ZAIATCHICK. M: SOTO. H. G.: JACOB. J. L. B.: BRAILE.

D. M. - Substituiçào completa da aorta ascendente e da valva aortica com tubo valvulado de pericardio bovino. Rev. Bras.

Cir. Cardiovasc. 2(2): 129-138. 1987.

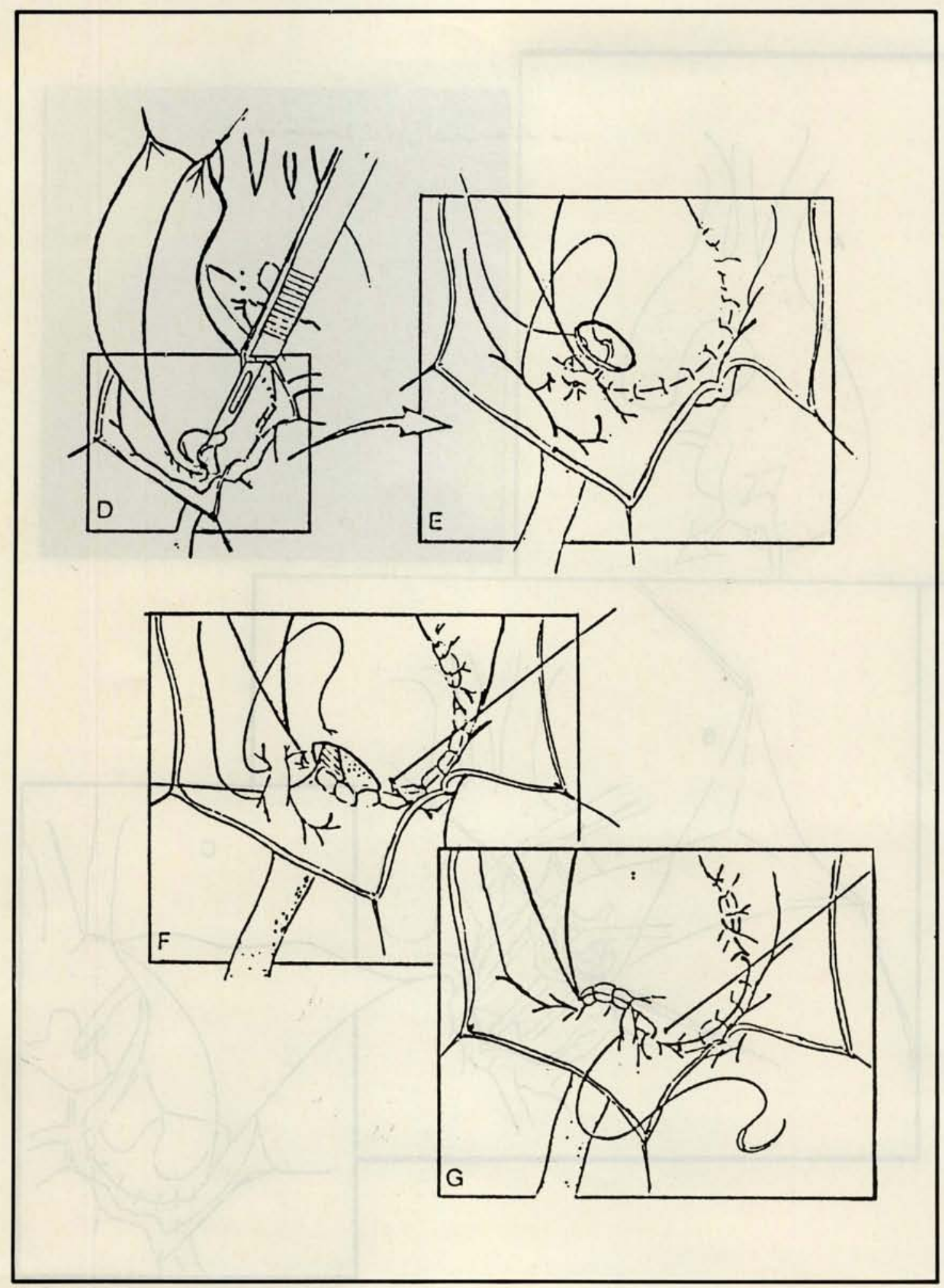

Fig. 1 - D, E, F, G 


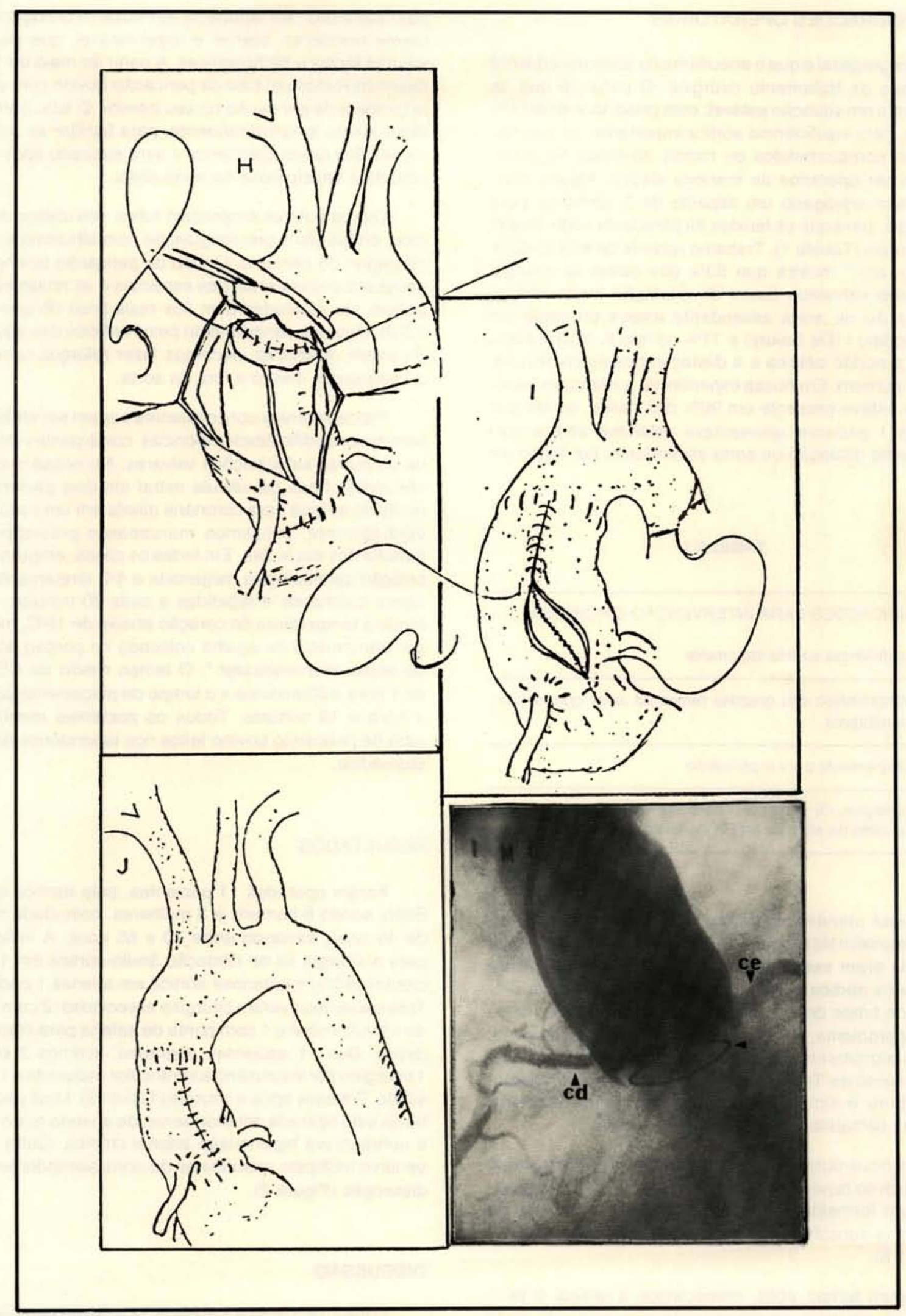

Fig. $1-\mathrm{H}, \mathrm{I}, \mathrm{J}$. 
ARDITO, R. V.: SANTOS. J. L. V.: MAYORQUIM. R. C.: GRECO. O. T: ZAIATCHICK. M.: SOTO. H. G.: JACOB. J. L. B.: BRAILE. D. M. - Substituição completa da aorta ascendente e da valva aortica com tubo valvulado de pericárdio bovino. Rev. Bras. Cir. CardiovasC. 2(2): 129-138, 1987.

\section{CONSIDERAÇŌES OPERATÓRIAS}

A regra geral é que o aneurisma da aorta ascendente necessita de tratamento cirúrgico. O paciente que se apresenta em situação estável, com pressão arterial controlada, sem insuficiência aórtica importante, ou que não estejam comprometidos os ramos da croça da aorta, podem ser operados de maneira eletiva. Alguns cirurgiōes têm advogado um aspecto de 3 semanas para a cirurgia, para que os tecidos da parede da aorta fiquem mais firmes (Tabela 1). Trabalho recente de KOUCHOUKOS et alii ${ }^{10}$ mostra que $53 \%$ dos casos de cirurgia com tubo valvulado foram de dilatação ânulo-aórtica; a dilatação de aorta ascendente esteve presente em $22 \%$ no tipo I (De Bakey) e $11 \%$ no tipo II, aparecendo, ainda, a aortite sifilitica e a dilatação pós-estenótica em menor número. Em nossa experiência, a dilatação ânuloaórtica esteve presente em $90 \%$ dos casos, sendo que apenas 1 paciente apresentava estenose aórtica com importante dilatação da aorta ascendente, por lesão em jato.

\section{TABELA 1}

\section{INDICAÇŌES PARA INTERVENÇÃO CIRÚRGICA}

- Insuficiência aórtica importante

- Compromisso dos grandes ramos da aorta com sinais neurológicos

- Sangramento para o pericárdio

- Processos de dissecção da aorta com dor continua e aumento da silhueta ao RX de tórax

Desta maneira, podemos fazer muitas considerações de ordem técnica. Primeiramente, as reconstruções da aorta eram executadas separadamente do implante da válvula aórtica estenótica ou insuficiência, com emprego de tubos de Dacron, acima das coronárias, onde o maior problema, apesar de pré-coagulação do enxerto, era o sangramento. Outras técnicas foram empregadas com enxerto de Teflon: retirada do aneurisma e anastomose pura e simples, com sutura contínua da íntima a outras camadas da aorta, com Prolene 4/0.

Em novembro de 1979, começamos a utilizar opatch de pericárdo bovino tratado com glutaraldeído e conservado em formaldeido, para o alargamento da raiz da aorta, nas substituiçōes valvares aórticas com anel estreito $(1,5)$.

Algum tempo após, começamos a utilizar o pericárdio bovino para as cirurgias de aneurisma da aorta ascendente, com melhora importante do sangramento pós-operatório, em virtude de ser material biológico altamente resistente, flexivel e impermeável, que permite suturas fáceis e hemostáticas. A partir de maio de 1985. desenvolvemos um tubo de pericárdio bovino com válvula também de pericárdio no seu interior. O tubo apresenta-se aberto longitudinalmente, para facilitar as anastomoses dos óstios coronários e será suturado após completada a anastomose na aorta distal.

Outros autores empregam tubos valvulados de Dacron, efetuando a pré-coagulaçāo com albumina e autoclavagem do conjunto. $\mathrm{O}$ tubo de pericárdio bovino nâo necessita dessas situaçōes especiais e as anastomoses podem ser efetuadas com fios mais finos (Prolene $4 / 0$ e 5/0), evitando sangramento pelos orifícios das agulhas. Se existir dissecção, podemos fazer reforços com tiras de pericárdio, dentro e fora da aorta.

Procedimentos concomitantes podem ser efetuados sem maiores dificuldades técnicas, como pontes de safena ou outras substituiçōes valvares. No nosso material, efetuamos trocá de válvula mitral em dois pacientes e ponte de safena para coronária direita em um caso. Preventivamente, utilizamos marcapasso provisório, na maioria dos pacientes. Em todos os casos, empregamos solução cardioplégica oxigenada a $4^{\circ} \mathrm{C}$ diretamente nos óstios coronários e repetidas a cada 20 minutos, mantendo a temperatura do coraçāo abaixo de $15^{\circ} \mathrm{C}$, medida por termômetro de agulha colocado na porção anterior do septo interventricular ${ }^{2}$. O tempo médio de CEC foi de 1 hora e 30 minutos e o tempo de pinçamento aórtico, 1 hora e 15 minutos. Todos os pacientes receberam tubo de pericárdio bovino feitos nos laboratórios da IMC Biomédica.

\section{RESULTADOS}

Foram operados 11 pacientes, pela técnica de De Bono, sendo 8 homens e 3 mulheres, com idade média de 46 anos, variando entre 20 e 65 anos. A indicação para a cirurgia foi de dilatação ânulo-aórtica em 10 pacientes $(90 \%)$ e estenose aórtica em apenas 1 paciente. Três pacientes tiveram cirurgias associadas: 2 com troca de válvula mitral e 1 com ponte de safena para coronária direita. Dos 11 pacientes operados, tivemos 2 óbitos, 1 cirúrgico por insuficiência ventricular esquerda e 1 óbito súbito, 7 meses após a cirurgia (Tabela 2). Uma paciente havia sido operada anteriormente, de enxerto aorto-renal e apresentava hipertensāo arterial crônica. Outra apresentava múltiplos aneurismas de aorta ascendente, sem dissecção (Figura 2).

\section{DISCUSSÃO}

O tubo valvulado é o método de escolha para pacientes com dilatação ânulo-aórtica, bem como para os pa- 
ARDITO, R. V.: SANTOS. J. L. V.: MAYORQUIM, R. C.: GRECO. O. T: ZAIATCHICK. M.: SOTO. H. G.: JACOB. J. L. B.: BRAILE.

D. M. - Substituição completa da aorta ascendente e da valva aórtica com tubo valvulado de pericárdio bovino. Rev. Bras. Cir. Cardiovasc., 2(2): 129-138, 1987.

TABELA 2

\begin{tabular}{|c|c|c|c|c|c|c|c|c|c|c|c|c|c|}
\hline NOME & SEX. & ID. & DIAGNOSTICO & T. PINC. & T. CEC. & $\begin{array}{l}\text { CIRURGIA } \\
\text { ASSOCIADA }\end{array}$ & M.P. & N: TUBO & $\begin{array}{l}\text { SANGUE } \\
\text { PLASMA } \\
\text { POS OP. }\end{array}$ & $\begin{array}{l}\text { REVISÁO } \\
\text { HEMOST. }\end{array}$ & RETORNO & HISTOLOGIA & OBITO \\
\hline E.A.M. & F. & 20 & $\begin{array}{l}\text { Aneu ao Asc. + } \\
\text { Insuf. Aörtica }\end{array}$ & 120 & 145 & - & V.D. & 23 & $\begin{array}{c}500 \mathrm{ml} \\
-\end{array}$ & - & 20.05 .86 & $\begin{array}{l}\text { Doença reumática } \\
\text { ativa - Endocard. }\end{array}$ & - \\
\hline D.P. & M. & 45 & $\begin{array}{l}\text { Aneu dissecante } \\
\text { de } A_{0}+\text { Insuf. } A_{0}\end{array}$ & $0^{105}$ & 133 & PS. Dir. & $\begin{array}{l}\text { A.D.e } \\
\text { V.E. }\end{array}$ & 25 & $\begin{array}{l}2.000 \mathrm{ml} \\
3.000 \mathrm{ml}\end{array}$ & - & - & $\begin{array}{l}\text { Doença reumatica } \\
\text { fibrose da intima }\end{array}$ & subito \\
\hline M.B.S. & M. & 65 & $\begin{array}{l}\text { Aneu Ao Asc. + } \\
\text { Insuf. Ao }\end{array}$ & 76 & 105 & - & V.D. & 29 & $1.100 \mathrm{ml}$ & - & 25.09 .86 & $\begin{array}{l}\text { Degeneraçảo mixo- } \\
\text { matosa da intima }\end{array}$ & - \\
\hline R.A. & M. & 61 & $\begin{array}{l}\text { Aneu Ao Asc. + } \\
\text { Insuf. Ao e M. }\end{array}$ & 90 & 120 & $\begin{array}{l}\text { T.V.M. } \\
\text { P.B. } n: 31\end{array}$ & V.D. & 29 & $\begin{array}{l}1.500 \mathrm{ml} \\
1.400 \mathrm{ml}\end{array}$ & 5.P.O. & 15.12 .86 & $\begin{array}{l}\text { Fibrose valvar } \\
\text { Ao e Mitral }\end{array}$ & - \\
\hline A.V. & M. & 41 & $\begin{array}{l}\text { Dilatação da Ao } \\
\text { Asc. + Insuf. Ao }\end{array}$ & 80 & 108 & - & - & 29 & $\begin{array}{l}600 \mathrm{ml} \\
500 \mathrm{ml}\end{array}$ & - & 26.09 .86 & $\begin{array}{l}\text { Degeneraçáo mixo. } \\
\text { matosa da Valva Ao }\end{array}$ & - \\
\hline V.N. & M. & 57 & $\begin{array}{l}\text { Aneu Ao Asc. + } \\
\text { Insuf. Ao }\end{array}$ & 113 & $155^{\circ}$ & $\begin{array}{l}\text { T.V.M. } \\
\text { P.B. n: } 31\end{array}$ & V.D. & 31 & $\begin{array}{l}300 \mathrm{ml} \\
250 \mathrm{ml}\end{array}$ & - & 14.10 .86 & $\begin{array}{l}\text { Fibrose da Aorta } \\
\text { e Mitral }\end{array}$ & - \\
\hline D.M.B. & F. & 41 & $\begin{array}{l}\text { Aneu Ao Asc. + } \\
\text { Insuf. AO }\end{array}$ & 80 & 115 & - & V.D. & 29 & $\begin{array}{l}500 \mathrm{ml} \\
500 \mathrm{ml}\end{array}$ & - & 14.10 .86 & $\begin{array}{l}\text { Degeneraçáo mixo. } \\
\text { matoxa da Valva Ao }\end{array}$ & - \\
\hline V.M.M. & F. & 53 & $\begin{array}{l}\text { Múltiplos Aneu } \\
\text { Ao Asc. + Ins. Ao }\end{array}$ & $0^{107}$ & 140 & - & V.D. & 25 & $\begin{array}{l}500 \mathrm{ml} \\
300 \mathrm{ml}\end{array}$ & - & 18.08 .86 & $\begin{array}{l}\text { Fibrose Valvar } \\
\text { Aórtica }\end{array}$ & - \\
\hline J.A.S & M. & 63 & $\begin{array}{l}\text { Aneu Ao Asc. + } \\
\text { Ao calcificada }\end{array}$ & + Est. ${ }_{105}$ & $135^{\prime}$ & - & - & 29 & $\begin{array}{r}1.000 \mathrm{ml} \\
500 \mathrm{ml}\end{array}$ & - & 14.01 .87 & $\begin{array}{l}\text { Fibrose + Calcif. } \\
\text { Valva aórtica }\end{array}$ & - \\
\hline M.M.M. & M. & 63 & $\begin{array}{l}\text { Aneu Dis. A0 + } \\
\text { Insuff. } A_{0}\end{array}$ & 113 & $170^{\circ}$ & - & V.D. & 29 & - & - & - & $\begin{array}{l}\text { Degeneração mixo. } \\
\text { mat. + Calcit. Valva Ao }\end{array}$ & $\begin{array}{l}\text { IV.E. + } \\
\text { I. Renal }\end{array}$ \\
\hline I.S. & M. & 35 & $\begin{array}{l}\text { Aneu Ao Asc. + } \\
\text { Insuf. Ao }\end{array}$ & $75^{\circ}$ & $100^{\circ}$ & - & - & 31 & - & - & 19.01 .87 & $\begin{array}{l}\text { Degeneraçăo mixo. } \\
\text { mat. + Calcifí. Valva Ao }\end{array}$ & - \\
\hline
\end{tabular}

cientes com dissecçāo aguda ou crônica de aorta, que apresentem dilataçāo valvar aórtica e incompetência.

A anastomose dos óstios coronários diretamente do tubo, ou a interposição de enxerto de veia não têm sido obstáculo técnico.

Vários autores sempre mencionam complicações com sangramento, sendo necessária a pré-coagulaçāo dos enxertos com albumina, sangue e trombina. Mesmo com estes cuidados, sempre é necessária a reposiçāo de grandes volumes de sangue no pós-operatório, além de plaquetas e fibrinogênio, o que nāo acontece com tubo valvulado de pericárdio bovino.

Já tivemos a oportunidade de reoperar pacientes em que fizemos alargamento da raiz da aorts e substituição valvar, nos quais notamos uma perfeita integração de patch, não havendo diferenciação do local ampliado com a aorta normal.
Os óbitos referidos não tiveram relação comio enxerto empregado, mas sim ao estado do miocárdio e na extensão da patologia do paciente.

A técnica de De Bono tornou a cirurgia da aorta ascendente de fácil execuçāo, encurtando, sobremaneira, o tempo operatório e melhorando, em muito, a sobrevida dos pacientes, no tempo intra e pós-operatório, como demonstrado por KOUCHOUKOS et alii ${ }^{10}$ (Figura 3).

Devemos salientar que o emprego do tubo valvulado de pericárdio bovino foi proposto quando já tínhamos usado o pericárdio bovino para fechamento de comunicação interventricular, comunicação interauiculai, dillpliação da via de saída do ventrículo direito na tetralogia de Fallot, reparo de lesões diafragmáticas, fechamento de pericárdio do paciente, apoio de suturas de bolsas de aorta, alargamento da aorta e istmoplastia na coarc- 
ARDITO, R. V.: SANTOS. J. L. V.: MAYORQUIM. R. C.: GRECO, O. T: ZAIATCHICK, M.: SOTO. H. G.: JACOB, J. L. B.: BRAILE. D. M. - Substituição completa da aorta ascendente e da valva aórtica com tubo valvulado de pericárdio bovino. Rev. Bras. Cir. Cardiovasc. 2(2): 129-138, 1987
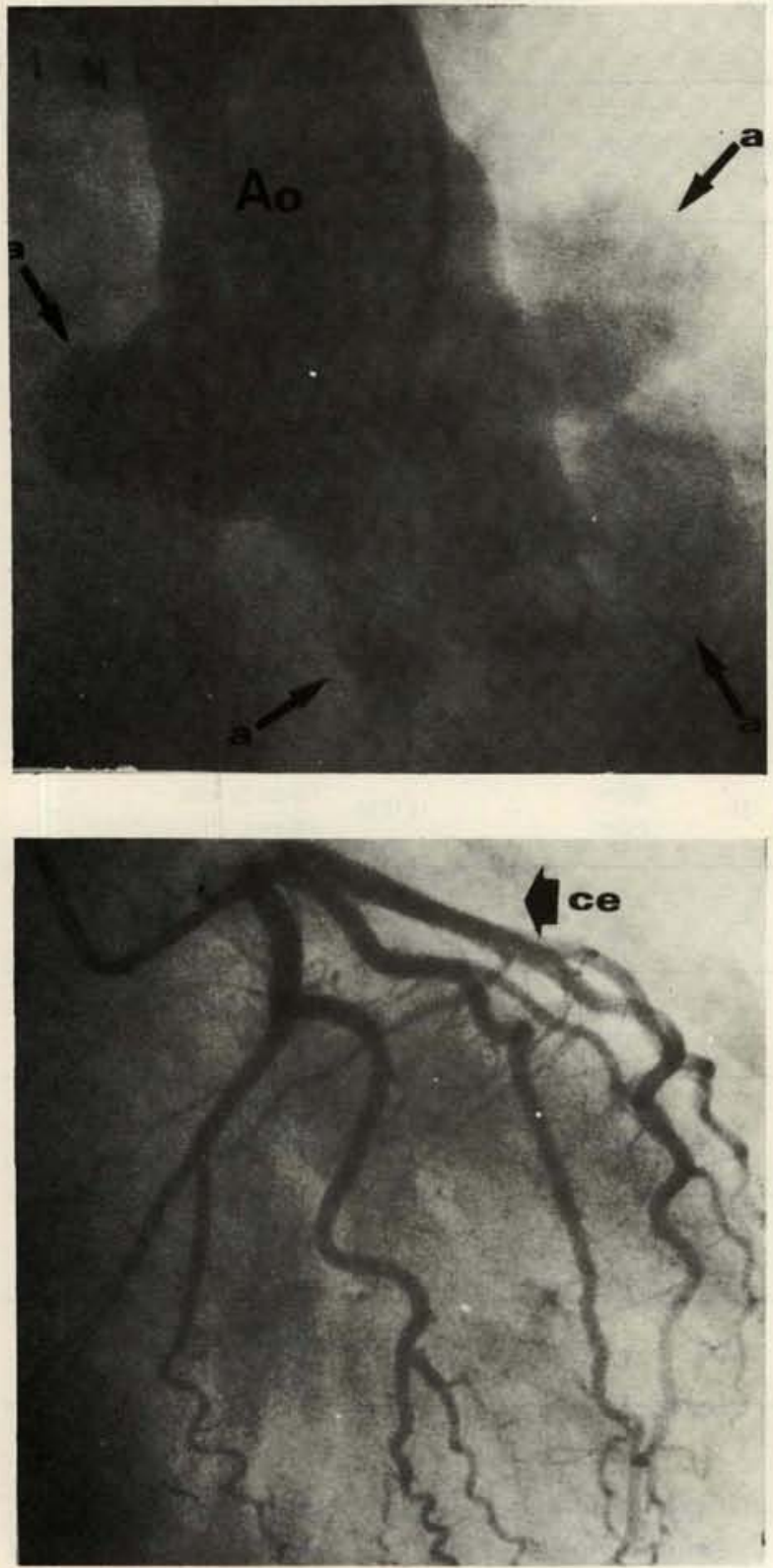

Fig. 2 - Múltiplos aneurismas na aorta.

tação da aorta ${ }^{3,6}$. Quanto à sobrevida destes pacientes, temos a referência de KOUCHOUKOS et alii ${ }^{10}$, que publicaram um acompanhamento de 11 anos, em pacientes que foram submetidos a cirurgia de aorta ascendente com tubo valvulado, apresentando uma sobrevida, em 127 pacientes, de $75 \%$ em 5 anos, $65 \%$ em 7 anos e $50 \%$ em 11 anos. Neste grupo, a ectasia ânulo-aórtica teve sobrevida de $80 \%$ em 5 anos e $70 \%$ em 7 anos o. disooco̧âo tovo sobrevida de $73 \%$ e $61 \%$ nestes períodos. As maiores causas de óbito foram: pseudo-aneurismas, endocardite, morte súbita, arritmias, infarto, insuficiencia renal e outras. O autor faz referência, também, de $11,8 \%$ de reoperaçōes por sangramentos, que
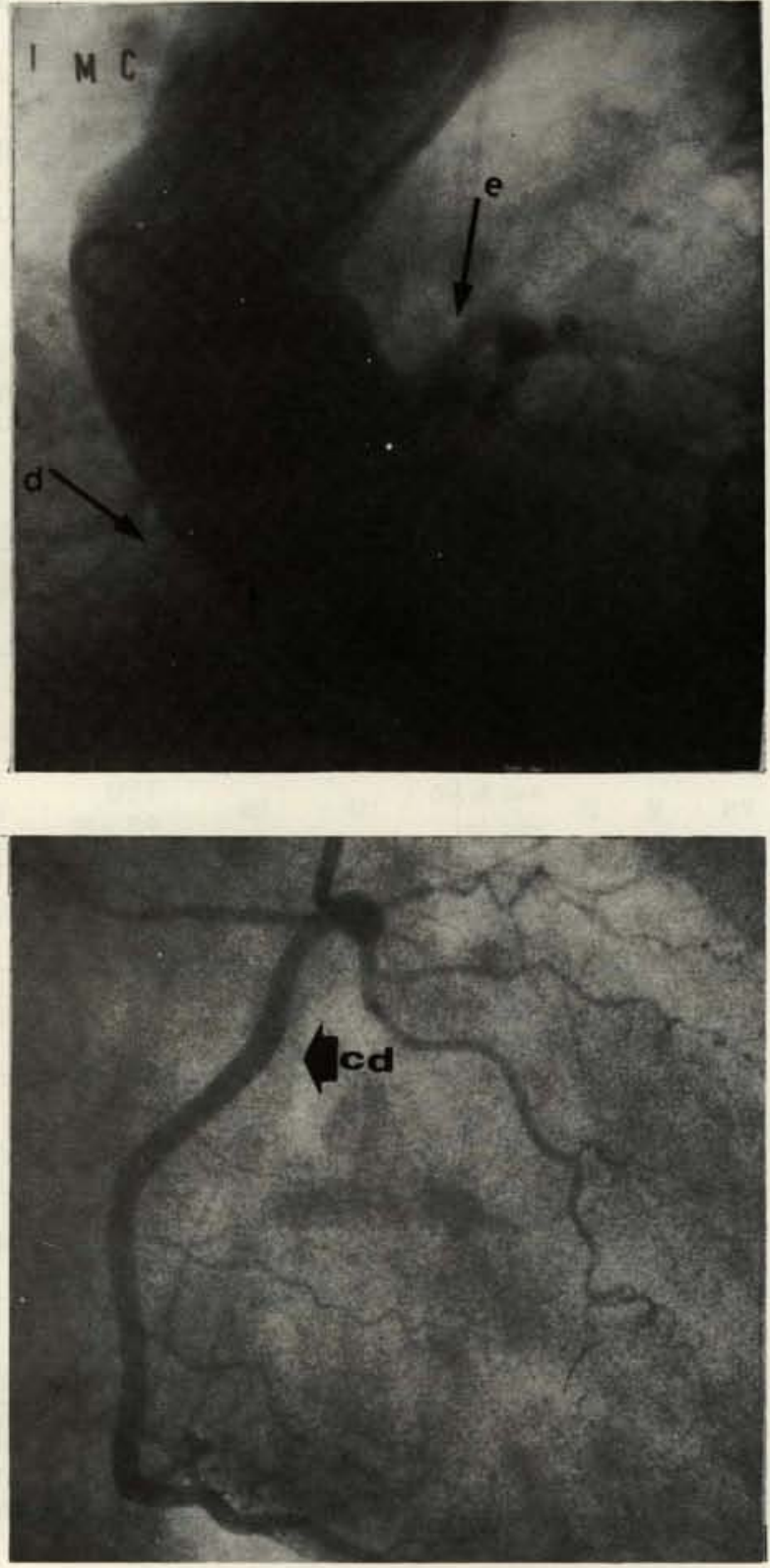

vieram a diminuir para $4,2 \%$, quando os enxertos começaram a ser pré-coagulados e autoclavados. No nosso material, tivemos apenas uma revisāo de hemostasia, por sangramento do enxerto e não devido ao enxerto.

Portanto, acreditamos que a técnica de enxerto valvulado é a cirurgia de escolha para as afecçōes da aorta ascendente, pela sua fácil execução, baixa mortalidade e com boa sobrevida tardia, e que o tubo, quando feito de pericárdio bovino, facilita ainda mais o ato operatório, pela sua alta flexibilidade e por ser altamente hemostático, diminuindo sobremaneira as reoperações por sangramento pós-operatório, não necessitando de medidas pré-coagulativas. 
ARDITO, R. V.: SANTOS. J. L. V.: MAYORQUIM, R. C.: GRECO. O. T: ZAIATCHICK. M.: SOTO. H. G.: JACOB. J. L. B.: BRAILE.

D. M. - Substituiçảo completa da aorta ascendente e da valva aórtica com tubo valvulado de pericardio bovino. Rev. Bras. Cir. Cardiovasc. 2(2): 129-138, 1987.
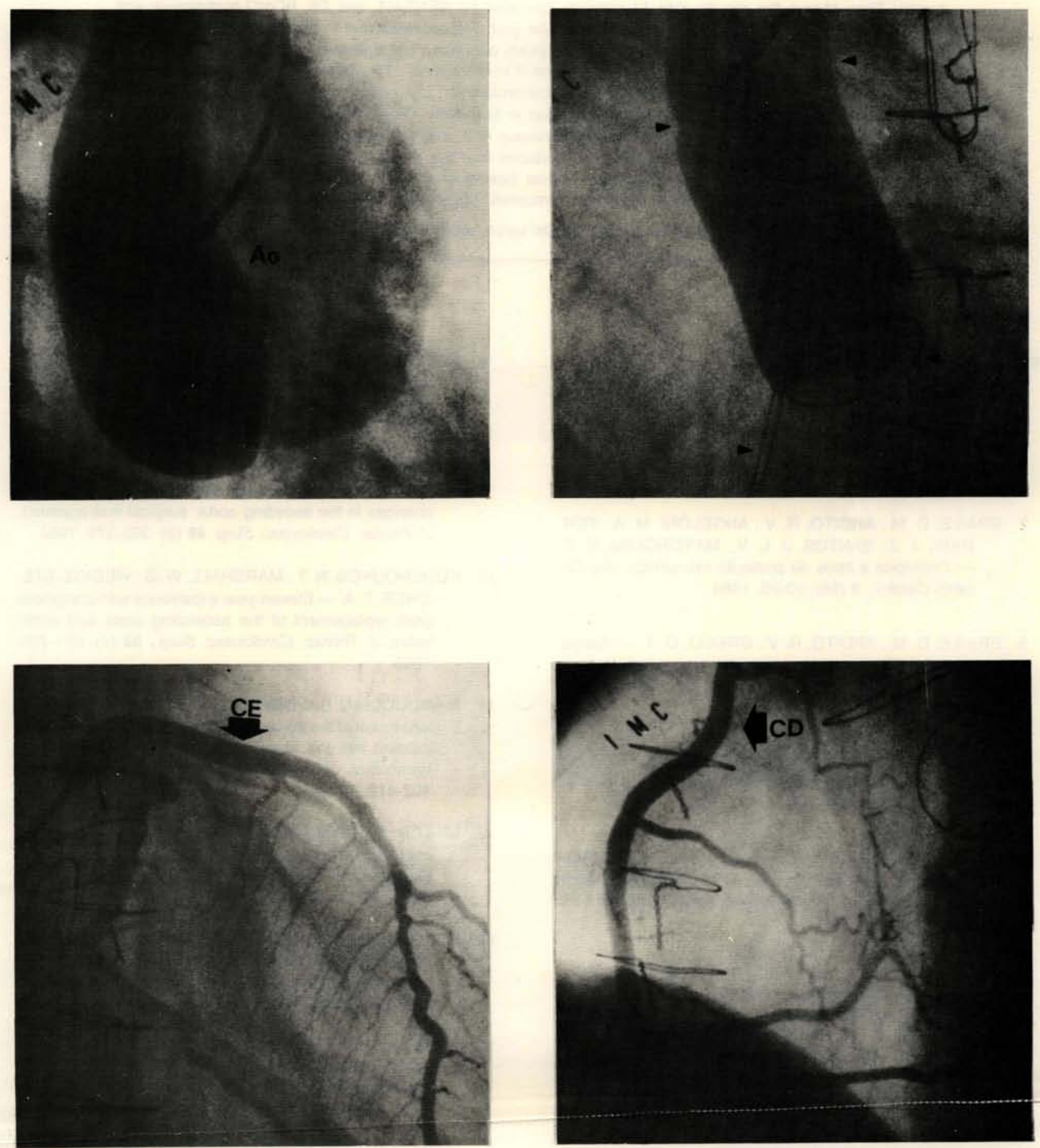

Fig. 3-Resultados cineangiográficos da cirurgia. 
ARDITO. R. V.: SANTOS, J. L. V.: MAYORQUIM, R. C.: GRECO. O T: ZAIATCHICK, M SOTO. H. G.: JACOB. J L. B: BRAILE. D. M. - Substituiçào completa da aorta ascendente e da valva aortica com tubo valvulado de pericardio bovino. Rev. Bras Cir. Cardiovasc. 2(2): 129-138, 1987.

RBCCV $44205-33$

ARDITO, R. V.; SANTOS, J. L. V.; MAYORQUIM, R. C.: GRECO, O. T.; ZAIANTCHICK, M.: SOTO. H

G.; JACOB, J. L. B.; BRAILE, D. M. - Total aortic valve and ascending aortic substitution with bovine pericardium valvar tube. Rev. Bras. Cir. Cardiovasc., 2 (2): 129-138, 1987.

ABSTRACT: The authors make a revision in the techniques for aortic aneurysm surgery with aortic valve lesion. They shown the results with 11 patients operated by BENTALL and DE BONO techniques with an open bovine pericardium IMC tubular and valvular graft without dead due to technic or due to the graft utilized. 8 patients, were male, age between $31-65$ years old (mean 50) 3 were female, age between 20-53 (mean 38); two surgeries were made in urgency and 9 in emergency. Two patients have associated mitral lesion associated with valvar change by IMC biological prosthesis. Just one patient needed a saphenous vein graft to right coronary artery because there was lesion in its ostium. The histopatologic showed mixomatosis degeneration in 4 cases, fibroses in 4 , reumatic disease in 2 , and pancarditis in 1 . After discourse about the method's complications and difficulties, we deduced that the thecnique employed is the choice from the treatment of this disease and pericardium valvar bovine graft turns the surgery more easy because it is flexible and hemostatic and doesn't need an hemostatic preparation before implantation.

DESCRIPTORS: ascending aorta, surgery; aortic valve, prosthesis; valvar tube graft.

\section{REFERÊNCIAS BIBLIOGRÁFICAS}

1 BENTALL, H. H. \& DE BONO, A. - A technique for complete replacement of the ascending aorta. Thorax, 23 (4): 338-339, 1968.

2 BRAILE, D. M.; ARDITO, R. V.; ANGELONI, M. A.; FERRARI, J. J.; SANTOS, J. L. V.; MAYORQUIM, R. C. - Principios e tipos de proteçăo miocárdica. Ars Curandi Cardiol., 8 (58): 23-26, 1986.

3 BRAILE, D. M.; ARDITO, R. V.; GRECO, O. T. - Alargamento da raiz da aorta com "patch" de pericárdio bovino preservado pelo glutaraldeído. Arq. Bras. Cardiol., 41 (4): 289-296, 1983.

4 COOLEY, D. A. \& De BAKEY, M. E. - Resection of entire ascending aorta in fusiform aneurysm using cardiac bypass. JAMA, 162 (12): 1158, 1956.

5 COOLEY, D. A.; BLOODWELL, R. D.; BEALL, A. C.; HALLMAN, G. L.; De BAKEY, M. E. - Surgical management of aneurysms of the ascending aorta. Surgical Clin. N. Am., 40: ( ): 1033-

6 CRAWFORD Jr., F. A.; SADE, R. M.; SPINALE, F. Bovine pericardium for conection of congenital heart defects. Ann. Thorac. Surg., 41 (6): 602-605, 1986.

7 CULLIFORD, A.T.; AYVALIOTIS, B.; SHEMIN, R. - Aneurysms of the ascending aorta and transverse arch. $J$. Ihorac. Candiovase. Surg., 83 (5): 701-710, 1982.
8 EDWARDS, W. S. \& KERR, A. R. - A safer technique for replacement of the entire ascending aorta and aortic valve. J. Thorac. Cardiovasc. Surg., 59 (1): 837-839, 1970

9 GROVES, L. K.; EFLER, D. B.; HAWK, W. A.; GULATI, K. - Aortic insufficiency secondary to aneurysmal changes in the asceding aorta: surgical management. J. Thorac. Cardiovasc. Surg. 48 (3): 362-379, 1964

10 KOUCHOUKOS, N. T.; MARSHALL, W. G.; WEDIGE-STECHER, T. A. - Eleven-year experience with composite graft replacement of the ascending aorta and aortic valve. J. Thorac. Cardiovasc. Surg., 92 (4): 691-705. 1986.

11 MANOUQUIAN, S. \& SEYBOLD-EPTING, W. - Patch enlargement of the aortic valve ring by extending the aortic incision into the anterior mitral leaflet: new operative technique. J. Thorac. Cardiovasc. Surg., 78 (3): 402-412, 1979.

12 MILLER, D. C.; STINSON, E. B.; OYER, P. E. - Concomitant resection of asceding aortic aneurysm and replacement of the aortic valve. J. Thorac. Cardiovasc. Surg., 79 (3): 388-401, 1980.

13 SINGH, M. P. \& BENTALL, H. H. - Complete replacement of the ascending aorta and the aortic valve for the treatment of aortic aneurysm. J. Thorac. Cardiovasc. Surg., 63 (2): 218-225, 1972.

14 ZUBIATE, P. \& KAY, J. H. - Surgical treatment of aneurysm of the ascending aorta with aortic insufficiency and marked displacement of the coronary ostia. J. Thorac. Cardiovasc. Surg., 71 (3): 415-421, 1976. 